and consequently difficult to tear. By these means the necessary laceration was made, without producing much strain on the ciliary attachments of the membrane; a good clear space became available for vision; and by the aid of a convex lens she was at once enabled to recognise the features of those around her.

In making a few comments on this case, I shall confine myself to a passing notice of its great physiological interest, its practical illustration of the value of modern means of investigating this class of diseases, and of the consequent precision with which they may be treated.

1. As the operation was performed while the hospital was in Charterhouse Square, now half a century ago, it affords the most remarkable instance with which I am acquainted of recovery of sight after so long an abeyance of the function; and it is not a little singular that the complicated nervous apparatus essential to vision should so long have maintained its integrity under circumstances so unfavourable.

2. It would be difficult to furnish a more pleasing illustration of the advantages that the ophthalmoscope has given us in arriving at a correct diagnosis, than this. Our predecessors ( to their lasting honour, be it said), by their genius and industry placed ophthalmology on a par with other cognate departments of medicine; but, Jacking the more exact means of physical diagnosis of our time, necessarily were obliged to speculate more or less on the hidden conditions of the eyeball, and, thus deducing their conclusions from inferential data, were without those landmarks that now may be observed in the progress or recession of disease. No longer left to speculate on subjective signs, we are enabled by the ophthalmoscope, etc., to seek for the physical conditions displayed by the interior of the eyeball. Nay, more im. proved means of research have made it incumbent on us to scrutinise with a precision not attained in any other branch of our science all functional diseases of the eye. Ten or twelve years have not sufficed to limit exactly or to daunt our hopes in the cure of blindness ; on the contrary, we have learned not to despair of vanquishing our foe till, at any rate, we have seen him, or, if invisible, till we have at least learned his habitat, and such other of his circumstances as may be within our ken; or, failing all these, till we have, per exclusionem, little doubt as to his power or our own inability to overcome him.

No doubt we have much to learn, much to see, much that, though seen, we are not yet able to interpret; still we have learned so much, seen so much, and interpreted so much, in the last decade, that we are daily becoming more tenacious, more hopeful, more ambitious, more daring. It certainly is true that we often obtain a glimpse of an enemy that makes us feel discretion to be the better part of valour. We have then, at least, the satisfaction of escaping the stigma of exhuusting ourselves on a hopeless contest. But, on the other hand, how often might the motto, "Ex fumo dare lucem", be our due; and, guided by the light of ophthalmoscopic exploration, we are enabled to descry the veil that shuts off light from an eye that, when unveiled, can see.

The Richmond Lunatic Asylum. The Dublin Corporation have this year rejected the presentment made for the sum of $£ 10,251$ for the Richmond Lunatic Asylum, on the ground that a number of lunatics from all parts of Ireland are there supplied at the expense of the Dublin citizens. They had sought in vain for a remedy, though the Government admitted the justice of their complaints. The tax for this object had increased $£ 7,000$ in the course of eleven years. The effect of the vote, however, is that the matter will go to the Court of Queen's Bench, which will issue its mandate for the collection of the $\mathfrak{f} 10,000$. Relief must be sought from the legislature to enable Boards of Guardians to send pauper lunatics to the districts in which they had resided a certain length of time.

\section{obrạinal Communitations.}

\section{FOREIGN OPINIONS OF THE NATURE OF}

\section{SYPHILIS.}

Collected by M. Berkeley HiLl, F.R.C.S., M.B.Lond.

III.-RoLlet of LyoNs.

M. Rollet is one of the earliest propounders of the theory of double contagion of venereal ulcers. This theory explains the apparently anomalous results and consequences of venereal infection, by referring their origin to two distinct contagious principles; the one, producing soft chancres, is a contagious pus, causing, by its irritating quality, ulcers where it is absorbed; the other is a true virus, which, like that of small-pox and of other diseases, affects the system generally, to the infection of which all the local symptoms are subordinate. To this virus alone are all the phenomena of constitutional syphilis referrible. In a pamphlet of seventy-eight pages, entitled De la Pluralité des Maladies Vénériennes, he has published his conviction of the distinct nature of the two ulcers in the year 1860; and from this the following extracts are made.

$\mathrm{He}$ first runs through the order of their appearance in history. Gonorrhœa is the oldest, being described by Moses, and by the Greeks, Arabs, and Romans.

The contagious ulcer of the genital organs, and its consequence, the inoculable bubo of the groin, appeared later. It is described as being widely spread and well known by the surgeons of the thirteenth and fourteenth centuries.

The syphilitic chancre was first observed at the end of the fifteenth century, when its appearance caused a terrible panic among the nations it invaded.

Hence, the doctrine of the plurality of venereal diseases is old ; and its present introduction to notice is simply a revival of a belief which was firmly held when syphilis was a new disease. Syphilis was well described until the two diseases were confounded together by Nicolas Massa in 1532 ; since which time others have followed his misleading, till M. Bassereau, in his treatise on Diseases of the Skin Symptomatic of Syphilis (Paris, 1852), rediscovered the distinct natures of venereal sores.

The points these diseases have in common are simply these. They are contagious; affect most frequently the genital organs; are usually transmitted by sexual intercourse. They often coexist in, and are often derived from the same sore. But all these latter characters are consequent on the first; namely, that they are contagious.

The sexual organs are the parts which fall into closest communication of all the parts of the body during the commonest mode of contact between individuals; viz., coitus. Rollet thinks it remarkable, not that these diseases have the sexual organs as a common seat, but that all contagious diseases do not select the genitals as the favourite locality. He believes that, if the acarus scabiei could exist in the vagina, it would be found there more often than anywhere else. Having discussed this question at some length, he passes on to arrange the points of difference of the two ulcers in five series.

First Series. Inoculation on the Patient Himself. (Auto-inoculability.) 'The contagious ulcer is almost indefinitely inoculable on its bearer. This experiment has been tried some thousands of times on the same individual, with never-failing success. For example, M. Lindmann inoculated himself 2200 times with soft chancrous pus without once failing. (See Ricord's Leçons sur le Chancre, page 335.) It is inoculable on syphilised 485 
and on unsyphilised persons alike. No disease but one completely local-as favus, for instance-can behave thus.

The indurated chancre can at no period be inoculated on its bearer. Experiments confirming this have been made again and again in all countries of Europe. Moreover, this ulcer is inoculable on no other person who is labouring under any form of constitutional syphilis.

The number of simple chancres is more often plural than single, through its power of repetition on the same individual; so that it repeats itself around the site of the original ulcer.

The almost complete absence of well authenticated cases of simple chancre caused by accidental contagion on the head and face and nipple, however it be explicable, is a peculiarity of this variety.

The infecting chancre is most usually single (perhaps all exceptions to this rule are " mixed chancres," which are explained later). It is most frequent on the genitals, nearly as frequent in the month, but has been observed on every part of the body's surface.

Second Series. Incubation and Mode of Development. The simple chancre has no incubation period. In twenty.four hours it is visible as a minute pustule; in four or five days it is a characteristic ecthymatous pus. tule, breaking down after that into a growing ulcer. The infecting chancre has a long period of inaction after in. oculation. In fifteen cases of artificial inoculation of syphilitic sores on persons virgin from syphilis, made by different observers, the shortest period was nine days and the longest forty-two; the mean being twenty-five days. Of these fifteen, three were inoculated from primary chancres, and twelve from secondary affections.

The simple sore commences as a pustule. When it has become an ulcer, it is pretty deep in form; it has borders which appears punched out perpendicularly, sometimes undermined; the floor is wormeaten, covered with greyish pus. 'The base is nearly always supple; when it is hard, its hardness is non-elastic and due to in. flammatory engorgement.

The infecting sore commences as a papale, which rapidly ulcerates; its ulcerated surface is coppery red or ham coloured; its borders are not sharply cut out; its sides slope down to the floor, which is smooth; the pus secreted on it is scanty, and often coagulates into a croupous layer or even into a crust.

The induration commences early, and steadily developes itself, presenting when developed a hard elastic base, feeling to the touch most frequently like a piece of parchment, or India-rubber, or gristle. This induration is frequently absent in women and even in men about once in twenty cases.

The transformation in situ of a primary lesion into a secondary one is more frequent in women than in men. A thin pellicle forms over the denuded surface of the ulcer, which thereby attains a papular appearance. This change, occurring so frequently in women, Rollet considers in some way to supply the want of induration of their sores.

Simple chancres are much more subject to sloughing, etc., than the infecting sore, owing to the superior irritating qualities of the former.

Third Series. Differences of the Consequences of the Two Sores. This, as remarked by Dr. Friedreich, his German critic, is what M. Rollet is busied with proving to be a result of the difference of the two sores; ergo, he rests his argument on a q.e.d. The sole consequence of the simple sore is an acute hubo, arising from absorption the next chain swells, grows very tender, and sharply painful; suppuration soon follows, of which the pus is plentiful, greyish, and inoculable, like that of the chancre. The opened abscess soon resembles the chancre in its sharply cut edges, ragged surface, and inoculable pus. This absorption does not always take place; and then there is only swelling and tenderness of the glands of the groin, as with any other cause of locak irritation. Here end the consequences of the simple chancre.

The infecting chancre causes enlargement of the groups of lymphatic glands generally; those nearest the sore being first affected and inevitably affected. The groups more distant from the sore are usually also enlarged. This enlargement is indolent, hard, and longo continuing; suppuration occurs now and then in thiss variety; but purely from irritation, and the pus is not inoculable. To this enlargement succeed secondaryo symptoms, at a more or less distant period. In the before mentioned fifteen cases, it appeared in the minimum period of twelve days, and in the maximum of $128 \overrightarrow{0}$ days, with a mean of fifty-two days.

Fourth Series. Differences in their Sources of Origin $\vec{\omega}$ The syphilitic chancre arises from inoculation, either of theo pus of a primary lesion, of the fluids of some secondary음 lesions, or of the blood of a person suffering some form? of secondary syphilis. Each of these sources producesio a chancre in the person infected. The semen can alsoge convey it to the ovum; and, in this instance, the pre-sence of a chancre is not necessary. In every other case, a chancre is the commencement of syphilis. The simple chancre results through contagion with the pus음 of the simple chancre.

These two laws have been proved by various observers, $\infty$ in addition to Rollet, Ricord, and Fournier, who have investigated a large number of cases where the persons in-क्ष fecting and the persons infected have been compared, and these comparisous have always established the simi larity and identity between the source and the propagated sore. Some of these cases of similarity were provedbefore the dualist doctrine was revived. For instance, when children have infected their nurses, the sores have? been long known to be hard chancres succeeded by philis. In twenty-four inoculations of syphilitic exura tions, the consequence has always been syphilis.

Fifth Series. Effects of Treatment. The simple sero can be eradicated at any period of its existence by cau-n terisation, which converts it into a simple suppuratingo wound, healing quickly, and no longer inoculable.

The infecting sore, though sometimes cauterisable with gond effect, cannot be destroyed so as to prevent constitutional results, with certainty, at any time, and without doubt. After the first few days, cauterisations are useless. Mercury is useless in preventing the progress of the simple sore; but it is universally acknowledged to be advantageous in the infecting sore.

In comparing the frequency of syphilis with other venereal diseases, Rollet makes use of statistics to bei quoted presently.

Coincidence of the Two Diseases on the same Indivi dual. The two diseases are often present together, because they are propagated in the same way; and they have their most frequent seat in common. This is onlyo a characteristic of contagious diseases generally. For instance, in inoculating vaccine matter, measles andN small-pox are sometimes inadvertently inoculated, being present along with the vaccine disease in the source of
infection.

The venereal diseases may exist, not only at once, butru in the same localities; so that the lesions they occasion have characteristics common to both sores. 'I'he syphi-c. litic poison may be inoculated; but, at the same time, $\sigma$ gonorrhœa and soft chancres muy be present, which completely mask the syphilitic sore until its secondary symp-cㅜㅇ toms show themselves elsewhere.

From the following statistics, Rollet thinks he can ? estimate the usual proportion of the cases of mixed dis- 0 eases to the cases of isolated disease. Of 2000 cases of venereal affections, gonorrhuea bore 41 per cent., simplexD chancres 33 per cent., infecting chancres 26 per cent. OAP these 2000 cases, 90 per cent. were uncomplicated withD 
each other; and 10 per cent. had either two or all three of these simultaneously present.

Mringled Cases. 1. A syphilitic patient without syphi. litic eruption on his genital organs has a gonorrhœe, which runs its usual course; if the pus alone be inserted in the mucous membrane of another, that second person has gonorrhœa only; if blood be taken with it, and the second be virgin from syphilis, he is infected with syphilis as well. The patient may have syphilitic affections of the genitals; in which case the syphilis and gonorrhœe may be transmitted simultaneously, and run their course independently of one another. If this syphilitic sore be an urethral chancre, the case would be very puzzling, as there would, perhaps, be no detectable sign of syphilis separate from the gonorrhœe. The point of this argument is, that blood of syphilised persons is inoculable, but their pus is not.

2. Simple chancres may coexist with infecting ones.

Mixed Chancres. A constitutionally syphilised individual contracts a simple chancre, which runs its usual course, except it may have greater tendency to pbagedæna. This chancre he may communicate to a non-syphilised individual as a simple chancre, and nothing more, if the contagious matter contain only pus and no blood. Should blood be mingled with the pus, this communicated sore will, in all probability, prove an infecting syphilitic sore. This chancre, in virtue of its mixed character, that of the simple and that of the infecting chancre, is inoculable on its bearer. (Rollet relates no case in support of this hypothesis.)

3. A person may bear both simple and infecting sores at the same time on different parts; but if they be pretty close together they may inoculate another indivi. dual at the same point, and so produce a sore having the characters of both. Or, again, a sore originally infecting may be inoculated with pus from a soft chancre; and then its characters would be mixed. The inoculation may take place in the reverse order. These combinations are rare, as the sores are usually separate.

M. Rollet concludes, from the results of artificial inoculation, that the "mixed chancre" holds a proportion of five per cent. to the infecting sore.

The mixed chancre, Rollet thinks, must have been described as the ulcus clevatum by older writers; its description so completely resembles that of the sore produced when the two contagious principles ure mixed.

From a few considerations on the coexistence of vaccine with syphilis, the following are extracted:-

If a syphilitic individual be vaccinated, whether he be new-born or adult, is immaterial. The irritation of the vaccine stimulates the action of the syphilitic poison; and syphilitic eruption appears earlier than it would otherwise have done. If, from this subject, vaccine pus without blood be taken, no syphilis will be communicated. This has been verified by different observers, whose experiments are narrated at length by $M$. Viennois, in a treatise on the Inoculation of Syphilis with Vaccination. (Archives Générales de Médecine, June, July, and Sept. 1860; and Gazette des Hôpitaux, March, April, and May 1862.) If blood of the syphilised person be taken with the pus of the vaccine, it is certain that syphilis will be infected. This question is treated at more length in the before-mentioned treatise.

The contagious principles of the venereal sores are two in number-the syphilitic poison and the contagious ulcer poison. The syphilitic virus is contained in the pus of the primary sore, the blood generally, and the serous tluid of secondary lesions. The spermatic fluid in a certain degree also contains it, as that fluid can communicate the disease to the ovum.

The contagious principle of the simple chancre is not sustained in any of the corporeal tiuids except the pus of the chancre and the bubo arising out of it.

This completes M. Rollet's exposé of his doctrines. It will be seen that he is a dualist in the most exact sense of the word; namely, that venereal ulcers arise from inoculation of two originally different contagious principles, which have and ever have had no relation with each other. There are other writers who incline to the view that the contagious principle of the simple ulcer was once a modification of the syphilitic virus, though now its effects are completely distinct from those produced by the syphilitic poison. 'These are, in this restricted sense, dualists also.

[To be continued.]

\section{đxamactions of 迶xamches.}

\section{BATH AND BRISTOL BRANCH.}

Case of sphacelus of the tongue.

By Augustin Prichard, Esq., Clifton.

[Read September 25th, 1862.]

George Nichols, aged 50, a coal-miner from Monmouth. sbire, was admitted as an in-patient on the 28th of last July. Ten months before that time, a small pimple appeared on the left side of his tongue, without any very obvious cause. $\mathrm{He}$ himself seemed inclined to assign it to his habit of smoking, which he had carried to excess, having, as he said, always held a pipe between his teeth, perpetually puffing whilst at his work underground. $\mathrm{He}$ was a sober man, and had never had syphilis.

The inflamed spot became worse, and so painful that he was unable to eat on that side of his mouth; and the disease increased so much for the next month, during which his tongue was daily touched with nitrate of silver, that he was unable to continue his occupation.

When I first saw him, his tongue was red, very hard and immovably fixed in its place behind the teeth ; but it was not at all swollen, nor ulcerated. His speech was very imperfect.

I was much at a loss to determine the nature of the disease; for, although the tongue was of a scirrhous hardness, the progress of the malady and the patient's freedom from any other signs of cancer or of glandular disease inclined me to consider it innocent, and not malignant induration.

I ordered him some iodide of potassium internally, good food, and a solution of the chlorate of potash with a small quantity of the tincture of the sesquichloride of iron for his mouth. There was no change in his state for a few days, but then he began to complain of increasing difficulty of swallowing; and the end of his tongue became very soft and dark, and ultimately it turned quite black. Hæmorrhage to a small extent, daily recurring, came on ; and with the blood, which was continually dribbling from his mouth, was mixed some black and excessively fotid matter.

He was directed to wash out his mouth continually with Condy's fluid diluted; and a little stimulus was given to him. The liquor carbonis detergens was also used, as well as many of the chlorides.

The disease spread until the whole of his tongue became a mass of soft and black putrifying tissue, which no disinfectant would touch. As might be supposed, his difficulty of swallowing became much greater, and he could get down but a few drops at a time; but he did not seem to be particularly affected by the mixture of the teaspoonful of wine and water or beef-tea with the putrid liquid oozing from the mass of sphacelus which nearly filled his mouth.

The poor fellow, throughout the progress of this horrible disease, always looked cheerful and hopeful and contented; and, although his speech was at this period very muffled and indistinct, we could understand him to say that, if he could swallow, he would be better. 